\title{
Fatores relacionados com tempo de internação prolongado em enfermaria de clínica médica
}

\author{
Factors related to prolonged hospitalization time in nursing medical clinic
}
Factores relacionados con la estancia hospitalaria prolongada en una guardería de clínica médica

Irineu Lopes de Alcântara Júnior ${ }^{1 *}$, Glauce Leão Lima ${ }^{1}$, Irilane de Alcântara Figueira², Ismael Henrique Azevedo de Alcântara ${ }^{2}$, Carlos Roberto Rohenkohl Evangelista Santos ${ }^{3}$, Isabella Marina Margel2 ${ }^{2}$ Angelo Bruno Pagoto ${ }^{3}$, Armando Hiroyuki Mori Júnior ${ }^{3}$, Bárbara Pires Ihara ${ }^{3}$, Talita Sampaio Carvalho3.

\section{RESUMO}

Objetivo: Avaliar os fatores que contribuem para o tempo de internação hospitalar dos pacientes da Enfermaria de Clínica Médica de um hospital da região metropolitana de um município do estado do Pará. Métodos: Realizou-se uma pesquisa quantitativa, transversal, do tipo descritiva e comparativa, com os dados provenientes de prontuários de pacientes com idade maior ou igual a 18 anos em regime de internação hospitalar nas enfermarias de Clínica Médica de um hospital em um município do estado do Pará, no período de janeiro a fevereiro de 2017. Resultados: Houve predomínio do sexo masculino e a faixa etária mais frequente foi de 40 a 59 anos, com média de idade de 54,2 anos e média do tempo de internação de 34,5 dias. Conclusão: Foi observado mais de um fator relacionado a um paciente, na qual a descompensação clínica da doença de base como complicações de cirrose hepática e Diabetes mellitus não especificado foram apontadas como os principais fatores responsáveis pelo aumento dos dias de internação.

Palavras-chave: Tempo de internação prolongada, Clínica médica, Tempo de internação hospitalar.

\begin{abstract}
Objective: To evaluate the factors that contribute to the length of hospital stay of patients in the Medical Clinic Ward of a hospital in the metropolitan region of a municipality in the state of Pará. Methods: A quantitative, cross-sectional, descriptive and comparative research was carried out, with data from medical records of patients aged 18 years or older undergoing hospitalization in the medical clinic wards of a hospital in a municipality in the state of Pará, from January to February 2017. Results: There were there was a predominance of males and the most frequent age group was 40 to 59 years old, with a mean age of 54.2 years and an average length of stay of 34.5 days. Conclusion: More than one factor related to a patient was observed, in which the clinical decompensation of the underlying disease such as complications of liver cirrhosis and unspecified Diabetes mellitus were identified as the main factors responsible for the increase in hospitalization days.
\end{abstract}

Keywords: Prolonged hospital stay, Medical clinic, Hospital stay.

\section{RESUMEN}

Objetivo: Evaluar los factores que contribuyen a la duración de la estancia hospitalaria de los pacientes en el Pabellón de la Clínica Médica de un hospital de la región metropolitana de un municipio del estado de Pará.

1 Universidade Federal do Pará (UFPA), Belém - PA. *E-mail: irineu.alcantara@gmail.com

2 Universidade do Estado do Pará (UEPA), Santarém - PA.

3 Universidade Federal do Amazonas (UFAM), Manaus - AM. 
Métodos: Investigación cuantitativa, transversal, descriptiva y comparativa. se realizó., con datos de las historias clínicas de pacientes de 18 años o más hospitalizados en las salas de la clínica médica de un hospital de un municipio del estado de Pará, de enero a febrero de 2017. Resultados: Hubo predominio de los varones y el grupo de edad más frecuente fue el de 40 a 59 años, con una edad media de 54,2 años y una estancia media de 34,5 días. Conclusión: Se observó más de un factor relacionado con un paciente, en el que la descompensación clínica de la enfermedad de base como las complicaciones de la cirrosis hepática y la Diabetes mellitus no especificada se identificaron como los principales factores responsables del aumento de los días de hospitalización.

Palabras clave: Estancia hospitalaria prolongada, Clínica médica, Estancia hospitalaria.

\section{INTRODUÇÃO}

Para recuperar a saúde de uma pessoa, um dos recursos a ser utilizado é a internação. A qualidade da assistência à saúde recebida por uma população é medida por estudos da taxa e do percentual de internação por causas evitáveis. Ademais, a atenção ambulatorial de boa cobertura e qualidade reduzem a taxa de internação. Adicionalmente, o usuário que fica no sistema de saúde também fica longe do convívio familiar e da comunidade, além de estar exposto a riscos evitáveis, como, por exemplo, infecção hospitalar (SILVA AMN $L F, 2014)$.

Conhecer fatores de risco é de suma importância para que se possa intrometer-se sobre os mesmos sendo de grande importância a constatação dos motivos que aumentam o período de internação, uma vez que são poucos os estudos na região e servirá como um alerta para se tomar medidas preventivas e até mesmo de conscientização de gestores de saúde e a própria população. Muitas vezes, fatores de risco em populações de hospitais terciários não são sobreponíveis a de hospitais secundários fora das grandes metrópoles. A qualidade da assistência à saúde é prejudicada diante do longo tempo de permanência hospitalar, relacionada às características e a procedência do paciente. Aumento de custo, morbidade e mortalidade são fatores que podem aparecer com o tempo de internação (BAHLIS LF, et al., 2014).

Os fatores que podem aumentar o tempo de internação hospitalar são diversos, variando de acordo com idade, sexo, assistência prestadas, diagnóstico e recursos disponíveis no hospital. Outro fator importante são as condições clínicas dos pacientes, o que pode gerar aumento das diárias. Quando relacionados com os processos da instituição e os recursos disponíveis, como, por exemplo, cancelamento ou atraso de cirurgias e atrasos na realização e entrega de resultados dos exames diagnósticos são passíveis de correção, sugerindo a necessidade de uma reformulação nos processos, para que eles fluam com maior eficiência e eficácia (SILVA AMN, 2014).

O aumento da expectativa de vida e, consequentemente, do numero de pessoas com mais de 60 anos ocasionaram mudanças no perfil da população e geraram demandas especificas de cuidado e atenção na área da saúde, com aumento da fragilidade biológica, psicológica e social (MOTTA CCR, et al., 2010). Com o passar da idade do paciente a internação é considerada de grande risco, pois o indivíduo idoso pode ter diminuição da capacidade funcional e, certas vezes, mudanças na qualidade de vida que podem ser irreversíveis (SILVA SA, et al., 2014).

É importante o reconhecimento das peculiaridades dos idosos durante o período em que estão internados no hospital, visando a identificação certa de problemas e a proposição de formas de atendimento diferenciadas que permitam melhor utilização dos recursos disponíveis e mais qualidade no atendimento7. Entre os fatores que podem vir a contribuir para o atraso da Alta Hospitalar (AH), está a dificuldade para prover os cuidados após a alta hospitalar, afinal, o Brasil possui graves problemas sociais, onde parte expressiva da população vive em condição de miséria e o acesso aos serviços de saúde é limitado (SILVA SA, et al., 2014).

A extrapolação do tempo de permanência pode levar à ocorrência de um aumento do custo hospitalar e diminuição da rotatividade dos leitos, o que nos mostra a necessidade de estudos que busquem cada vez reduzir a ocorrência desses motivos, que geram a extrapolação do tempo de permanência. Coligado a isso, 
a utilização de protocolos específicos pode definir a melhora na morbidade por meio do planejamento da assistência prestada ao indivíduo (SILVA AMN, 2014). A hospitalização prolongada também está associada ao risco aumentado de ser acometido por uma intempere associada à internação hospitalar, como a administração errada de medicamentos, a aquisição de uma infecção relacionada à assistência em saúde, a ocorrência de úlceras de pressão, aumento do risco de queda e ocorrência aumentada de trombose venosa profunda (SILVA RF, et al., 2018).

Além do alto custo relacionado à hospitalização prolongada, é considerável levar em conta a qualidade de vida dos acompanhantes e cuidadores, que estão em sobrecarga psicológica e laboral elevadas. A sobrecarga emocional e laboral em que os pacientes, acompanhantes e cuidadores são submetidos aumentam devido ao fato de serem uma população carente social e economicamente, com pouca oferta de lazer e contando quase que exclusivamente com a rede de apoio terciária quando hospitalizados, diminuindo consideravelmente a qualidade de vida durante a internação hospitalar (SILVA RF, et al., 2018).

O objetivo do estudo foi avaliar os fatores que contribuem para o tempo de internação hospitalar dos pacientes da Enfermaria de Clínica Médica de um hospital da região metropolitana de um município do estado do Para.

\section{MÉTODOS}

O estudo apresentou - se como uma pesquisa quantitativa, transversal, do tipo descritiva e comparativa. Os dados foram coletados durante o período de internação hospitalar nas enfermarias de Clínica Médica de um hospital universitário em uma cidade do Para, no período de 01/01/2017 até 28/02/2017.

Foram selecionados como participantes da pesquisa todos os pacientes com idade maior ou igual a 18 anos, que estavam em regime de internação hospitalar na enfermaria da clínica médica de um hospital universitário no estado do Para, no período referido.

Foram analisados prontuários de pacientes, sendo feito o preenchimento de um protocolo de pesquisa visando-se obter informações clínicas e epidemiológicas tais como sexo, idade, motivo da internação, tempo de internação, fatores relacionados com o tempo de internação prolongada e evolução para alta ou óbito.

Após essa primeira análise, foi feita a verificação de pacientes que ultrapassaram o tempo estipulado de diária de permanência maior, classificando-os como internação prolongada ou não. A classificação utilizada no presente estudo é baseada na tabela de acordo com o Sistema de Gerenciamento de Tabela e Processamentos (SIGTAP) disponível pelo Ministério da Saúde no Datasus (http://sigtap.datasus.gov.br/tabela-unificada/app/download.jsp).

Os dados foram obtidos em valores numéricos passíveis de análise estatística. Os dados foram apresentados de forma descritiva através de dados de média aritmética. Por fim, estes foram analisados no programa Excel, componente do Microsoft Office $\AA$ (versão 2013), para Windows $\AA$, onde foram organizados e transformados em tabelas.

Esta pesquisa foi realizada conforme os aspectos éticos da resolução 466/2012 do Conselho Nacional de Saúde (CNS), após a aprovação e submissão ao Comitê de Ética em Pesquisa (CEP) da UFPA - Hospital Universitário João de Barros Barreto da Universidade Federal do Pará, CAAE: 79910917.3.0000.0017. Neste estudo, os sujeitos foram codificados para garantir o anonimato. Os dados que não estavam completos não foram contabilizados na população final utilizada na análise de dados.

\section{RESULTADOS}

Durante a pesquisa foram colhidos os prontuários dos pacientes que tiveram alta no período de janeiro e fevereiro de 2017. Ao todo foram 112 os pacientes que tiveram alta, sendo que 90 pacientes foram excluídos do protocolo de estudo devido seus prontuários não serem encontrados (principal fator) ou terem informações incompletas nos prontuários solicitados (Nome, Número da matrícula, Sexo, Idade, Fator (es) relacionado com o tempo de internação prolongada e Tempo de internação), totalizando no final 22 pacientes que foram estudados. 
No que diz respeito sobre os dados epidemiológicos, houve um predomínio do sexo masculino $(14 ; 63$, $64 \%$ ) em relação ao feminino ( $8 ; 36,36 \%$ ). A média de idade dos pacientes pesquisados foi de 54,2 anos e a média do tempo de internação dos pacientes com o tempo de internação prolongada foi de 34,5 dias. A faixa etária mais frequente dos pacientes foi de 40 a 59 anos, representando $36,4 \%$ do total de pacientes (Tabela 1).

Tabela 1 - Distribuição dos pacientes com diárias extrapoladas, internados na enfermaria de clínica médica do hospital universitário.

\begin{tabular}{ccc}
\hline Faixa etária & $\mathbf{N}$ & $\%$ \\
\hline $\mathbf{2 0}$ a 39 anos & 5 & 22,7 \\
$\mathbf{4 0}$ a 59 anos & 8 & 36,4 \\
$\mathbf{6 0}$ a 79 anos & 5 & 22,7 \\
$\mathbf{8 0}$ ou mais anos & 4 & 18,2 \\
\hline Total & 22 & 100 \\
\hline
\end{tabular}

Fonte: Alcântara Júnior IL, et al., 2021.

Com relação aos fatores relacionados ao tempo de internação, foi observado mais de um fator relacionado a um paciente, na qual a descompensação clínica da doença de base, tendo como principais frequências: as formas de cirrose, as quais foram apontadas como principais contribuintes para aumento dos dias de internação, seguida da Diabetes mellitus não especificado com complicações. Outra questão importante foi que pacientes infectados acabaram preenchendo os critérios de internação hospitalar prolongada para serem submetidos à antibioticoterapia devido intercorrências infecciosas (Tabela 2).

Além das características clínicas já relatadas, no presente estudo foi observado que alguns pacientes apresentaram fatores administrativos que prolongaram o tempo de internação hospitalar, como aguardo para a realização de exames laboratoriais $(n=11)$ e de imagem $(n=10)$. Os fatores que menos se relacionam com o tempo de internação prolongada incluem: aguardo de medicação, atraso na avaliação de especialidade médica solicitada e aguardo de vaga em hospital referenciado (Tabela 2).

Tabela 2 - Fatores Relacionados ao tempo de internação na enfermaria de clínica médica do hospital universitário.

\begin{tabular}{ccc}
\hline Fatores relacionados ao tempo de internação & N & $\%$ \\
\hline Descompensação clínica do quadro de base & 21 & 95,4 \\
Atraso no resultado de exames laboratoriais & 11 & 50 \\
Término de antibioticoterapia & 10 & 45,4 \\
Atraso na realização ou laudo de exames de imagem invasiva & 10 & 45,4 \\
Atraso na realização ou laudo de exames de imagem não invasiva & 5 & 22,7 \\
Aguardo de procedimento médico cirúrgico & 2 & 9,1 \\
Aguardo da medicação & 1 & 4,5 \\
Atraso na avaliação de especialidade médica solicitada & 1 & 4,5 \\
Aguardo de vaga em hospital referenciado & 1 & 4,5 \\
\hline
\end{tabular}

Fonte: Alcântara Júnior IL, et al., 2021.

Dentre os principais diagnósticos observados nos pacientes internados durante o período da pesquisa, os dados coletados demonstram que existe uma prevalência de pacientes diagnosticados com alguma doença hepática $(n=8$, Outras formas de cirrose, Cirrose hepática alcóolica e Doença hepática sem outra especificação) e diabetes mellitus não especificado com complicações $(n=5)$, enquanto, dor localizada em partes do abdome, gastroenterite e colite não infecciosa não especificada, outras colites ulceradas, outras doenças do estômago e duodeno e lúpus eritematoso sistêmico foram diagnósticos menos encontrados (Tabela 3). 
Tabela 3 - Principais diagnósticos de alta observados na enfermaria da clínica médica do hospital universitário.

\begin{tabular}{ccc}
\hline Diagnósticos de alta & N & $\%$ \\
\hline Outras formas de cirrose & 4 & 18,1 \\
Cirrose hepática alcóolica & 3 & 13,6 \\
Doença hepática sem outra especificação & 1 & 4,5 \\
Diabetes mellitus não especificado com complicações & 5 & 22,7 \\
Hemorragias gastrointestinais sem outras especificações & 2 & 9,9 \\
Outras formas de tuberculose & 2 & 9,9 \\
Dor localizada em partes do abdome & 1 & 4,5 \\
Gastroenterite e colite não infecciosa não especificada & 1 & 4,5 \\
Outras colites ulceradas & 1 & 4,5 \\
Outras doenças do estômago e duodeno & 1 & 4,5 \\
Lúpus eritematoso sistêmico com comprometimento de órgãos e & 1 & 4,5 \\
sistemas & & \\
\hline
\end{tabular}

Fonte: Alcântara Júnior IL, et al., 2021.

\section{DISCUSSÃO}

Acerca dessa temática, um estudo realizado no ano de 2012 foi constituído por uma amostra de 48 pacientes, onde a idade dos participantes variou de 18 a 85 anos, com média de 50 anos, tendo predomínio do sexo feminino e a média do tempo de internação obtida foi de 20,9 dias (RUFINO GP, et al., 2012). Já no estudo de Motta CCR, et al. (2010), a faixa etária de maior prevalência foi de 70 a 79 anos. O presente estudo identificou maior prevalência de pacientes do sexo masculino internados, indo de encontro com os resultados de Rufino GP, et al. (2012). O tempo médio de permanência no presente estudo esteve muito acima da média de outros estudos.

O estudo de Cordeiro RLR, et al. (2016) também evidencia uma prevalência do sexo feminino, com $56 \%$ dos pacientes internados, assim como o estudo de Siqueira AB, et al. (2004) onde $54 \%$ eram do sexo feminino. O tempo médio de permanência em enfermaria foi de 16 dias, com $69 \%$ dos pacientes recebendo alta hospitalar como desfecho final.

Os índices de internações hospitalares entre as diversas faixas etárias apresentam números semelhantes, enquanto nesta pesquisa a faixa etária mais frequente foi de 40 a 59 anos (LIMA, et al., 2013). O estudo de Lima SBS, et al. (2013) teve maior semelhança com o presente estudo em relação à faixa etária. O registro dessa faixa etária semelhante deve se dar talvez devido ao perfil hospitalar de internações de pacientes cirróticos (TIENSOLI SB, et al., 2014).

Analisando os dados obtidos por essa pesquisa, também foi possível verificar que a faixa etária que oscila de 60 a 79 anos e representa a população idosa estudada também merece destaque (RODRIGUES CC e RIBEIRO RCHM, 2012). Esse grupo é considerado como mais fragilizado e suscetível a diversidade de desfechos clínicos, sendo necessário cuidados específicos e atenção diferenciada (CASTRO VC, et al., 2013). Nesse sentido, é possível inferir que o número elevado de idosos internados durante a elaboração desse estudo deve-se a essa vulnerabilidade fisiológica encontrada nesse segmento populacional (CORDEIRO LRL, et al., 2016).

Um estudo que buscou verificar quais fatores que estão relacionados com o período de internação apresentou o seguinte resultado: cultura positiva por germe multirresistente, internação clínica com necessidade de procedimento médico cirúrgico, utilização de antibioticoterapia sendo este último o que possui um dos maiores índices em ambos os estudos e presentes também nessa pesquisa (BAHLIS LF, et al., 2014). Muitas vezes, associado a necessidade de manter o paciente internado para término dos dias de administração de antibioticoterapia apesar de melhora clínica, não desmerecendo a necessidade de manutenção de internação nesses casos já que muitas das vezes se torna impraticável manter tais antibióticos em regime domiciliar devido características próprias dos mesmos. 
A permanência hospitalar prolongada (PHP) é um dos indicadores da qualidade do cuidado prestado aos pacientes nos serviços terciários, de forma indireta no caso. A respeito do tempo que tiveram que esperar por uma vaga na clínica médica existe a duração média das internações. Tal fato, além de diminuir a rotatividade dos leitos e aumentar os custos hospitalares, aumenta o risco de complicações decorrentes da internação, como as infecções hospitalares (RUFINO GP, et al., 2012).

O estudo de Cordeiro RLR, et al. (2016) evidenciou que o tempo de internação prolongada está associado a maior mortalidade, não estando associado à idade, escolaridade ou estado civil e escores elevados em escalas de avaliação funcional. Cordeiro RLR, et al. (2016) também evidencia que quanto maior a dependência funcional, maior o risco de hospitalização prolongada e ressalta que fatores como a presença de delirium, comprometimento das atividades da vida diária e múltiplas comorbidades são determinantes para uma mortalidade maior.

O estudo de Maues CR, et al. (2007), que objetivou analisar a epidemiologia dos idosos internados em enfermaria de Clínica Médica, alerta para o perigo de internação prolongada para idosos. Em seu trabalho a principal causa de internação foi acidente vascular cerebral, a sondagem vesical o procedimento mais realizado, infecção do trato urinário a intercorrência mais observada e o tempo de permanência hospitalar foi prolongado. $O$ estudo de Siqueira AB, et al., (2004) destaca as doenças cardiovasculares como maior causa de internação, seguida pelos transtornos mentais e doenças do aparelho respiratório. Essas causas de internação estão relacionadas a pacientes com idade mais avançada.

Além da idade, o tempo de internação em idosos pode acarretar em infecções hospitalares causadas por microrganismos multirresistentes. Ferraz CCB, et al. (2016) em sua pesquisa objetivou conhecer os fatores associados às infecções hospitalares causadas por microrganismos multirresistentes num hospital de ensino de Campo Grande/MS no ano 2012 e entre os 110 pacientes mais de $68 \%$ dos pacientes era sexo masculino e cerca de $42,7 \%$ tinha idade igual ou superior a 60 anos.

Conhecer fatores de risco é fundamental para que se possa intervir sobre os mesmos (BAHLIS LF, et al., 2014), sendo de grande importância a constatação dos motivos que aumentam o período de internação, uma vez que são poucos os estudos na região e servirá como um alerta para se tomar medidas preventivas e até mesmo de conscientização de gestores de saúde e a própria população.

Uma das limitações dos estudos atuais referentes a esse tema, segundo Silva AMN (2014), é que esses estudos são realizados em uma única instituição hospitalar, o que acaba impedindo dessa forma correlações entre hospitais de mesmo porte. Outras limitações são os registros em prontuários, características da população assistida e dos profissionais envolvidos, por exemplo.

Castro VC, et al. (2014) dizem que vários fatores favorecem a realização de estudos com base em dados do SUS, principalmente pela possibilidade de supor que o quadro de morbidade hospitalar brasileira mostrado por esse sistema se aproxime da realidade, visto que as taxas de cobertura do sistema de internações no Brasil são elevadas. Eles ressaltam que alguns dados podem apresentar restrições como, por exemplo, a disponibilidade de dados referentes somente às internações custeadas pelo SUS. Williams TA, et al. (2005) relatam, em seu trabalho onde realizaram revisão sistemática de literatura, que a

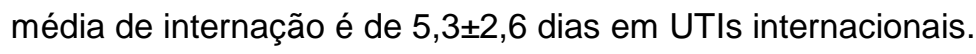

Os agentes que corroboraram para o atraso na alta hospitalar são: espera para realização de exames complementares e aspectos atrelados à responsabilidade médica (SILVA SA, et al., 2014), bem como o atraso na realização de exames que também está presente nesta investigação (BAHLIS LF, et al., 2014; SILVA AS, et al., 2014). Entretanto, é necessário ressaltar que existe diferença nos locais de pesquisa estudos, onde um foi realizado na clínica cirúrgica enquanto outro em uma clínica médica.

Os fatores que mais influenciaram no período de internação são: espera para a realização de exames complementares, atrasos na liberação dos laudos e exames e dificuldade de interconsultas (SILVA LF, et al., 2014). Neste estudo, apesar de achados estatísticos ligeiramente diferentes verificasse que a desestabilização clínica do quadro de base, o término da antibioticoterapia e o atraso na realização de exames foram os principais responsáveis pelo acréscimo de tempo de permanência hospitalar, exercendo influência 
no aumento da assistência ao paciente e no período prologado de internação. No estudo de Siqueira $A B$, et al. (2004), as principais complicações ocorridas nos pacientes foram rebaixamento do nível de consciência, broncopneumonia, ulcera por pressão, desidratação, infecção do trato urinário e insuficiência respiratória, ocasionando em um tempo maior de internação hospitalar.

O hospital em que foi realizada a pesquisa é referência em doenças infectocontagiosas e em pacientes hepatopatas, logo, essa circunstância, pode explicar os achados relativos a cirrose, problemas gastrointestinais e tuberculose. Além disso, essa instituição é considerada referência estadual em endocrinologia e diabetes, fato que também repercutiu nos resultados encontrados neste estudo. A pneumonia foi causa importante de internação hospitalar prolongada e resultou em uma média de 8 dias de internação hospitalar (MOLNAR S, et al., 2005; GARAU J, et al., 2008; GARCIA-VIDAL C, et al., 2009).

Os pacientes com diabetes mellitus são internados com maior frequência que a população em geral, sendo alta a prevalência de readmissão hospitalar desses indivíduos (RUFINO GP, et al., 2012). É possível afirmar, que esses dados concordam com o apresentado na presente investigação científica, na qual o diagnóstico por diabetes mellitus representa boa parte dos casos encontrados. Além disso, diversos estudos apontam o risco nutricional elevado nos pacientes internados, principalmente idosos, dificultando a resolução clínica e a alta hospitalar (CRESTANI N, et al., 2011; CRUZ LB, et al., 2012; MARTINS RCFC, 2018).

Em 2014 dois estudos sobre o tema foram publicados, em um deles os diagnósticos mais encontrados nos pacientes durante a investigação foram de causas neurológicas, seguidas por doenças cardíacas (TIENSOLI SB, et al., 2014). Já no outro trabalho, foi observado que os diagnósticos mais encontrados foram relativos a doenças respiratórias, doenças do sistema cardiovascular e patologias gastrointestinais (MORAES A, et al., 2014). As patologias mais associadas foram diferentes em relação ao presente estudo, mas isso pode ser explicado pelo perfil hospitalar dos pacientes (SOUSA BA, et al., 2011).

\section{CONCLUSÃO}

Esta pesquisa apresentou predomínio do sexo masculino, com faixa etária mais frequente de 40 a 59 anos e média do tempo de internação de 34,5 dias. Concluiu-se que, a descompensação clínica do quadro de base proporcionada principalmente por alguma das formas de cirrose, seguida do diabetes mellitus com complicações, foram apontadas como os principais fatores responsáveis pelo aumento do tempo de internação nesse período, seguidos de infecções e atraso na realização de exames. Como limitações do estudo, tivemos o tamanho da amostra reduzida principalmente pela ausência de prontuários. Tais fatores sugerem a possibilidade da criação de prontuários digitalizados ou um aplicativo que possa manter um banco de dados para facilitar a busca por prontuários ou algo semelhante.

\section{REFERÊNCIAS}

1. BAHLIS LF, et al. Fatores associados à internação prolongada em pacientes clínicos em hospital secundário do interior do Brasil. p. 89 . In:. São Paulo: Blucher, 2014.

2. CASTRO VC, et al. Perfil de internações hospitalares de idosos no âmbito do sistema único de saúde. Rev Rene, 2013; 14(4): 791-800.

3. CORDEIRO RLR, et al. Fatores relacionados ao óbito e à internação prolongada em uma enfermaria de geriatria. Geriatr Gerontol Aging, 2016; 10(3): 146-150.

4. CRESTANI N, et al. Perfil nutricional de pacientes adultos e idosos admitidos em um hospital universitário. Revista Ciência e Saúde, 2011; 4(2): 45-49.

5. CRUZ LB, et al. Perfil antropométrico de pacientes internados em um hospital universitário. Rev HCPA, 2012, 32(2): 177-181.

6. FERRAZ CCB, et al. Fatores associados a infecções hospitalares causadas por microorganismos resistentes em um hospital de ensino. PECIBES, 2016; 2(1): 52:57.

7. GARAU J, et al. Factors impacting on length of stay and mortality of community-acquired pneumonia. Clin Microbiol Infect, 2008; 14(4): 322-329.

8. GARCIA-VIDAL C, et al. Factors associated with prolonged hospital stay in community-acquired pneumonia. Enf Infecc Microbiol Clin, 2009; 27(3): 160-164.

9. LIMA SBS, et al. Perfil clínico-epidemiológico dos pacientes internados no pronto-socorro de um hospital universitário. Revista Saúde Santa Maria, 2013; 39(1): 77-86. 
10. MARTINS RCFC. Perfil nutricional, tempo de permanência e desfecho clínico de pacientes internados em unidade de terapia intensiva em um hospital filantropo de Ouro Preto - MG. Dissertação (Mestrado em Saúde e Nutrição) Escola de Nutrição. Universidade Federal de Ouro Preto. Ouro Preto, 2018; 108 p.

11. MAUES CR. Epidemiologia de idosos internados na enfermaria de clínica médica de hospital público. Revista Paraense de Medicina, 2007; 21(3): 31-36

12. MOLNAR S, et al. Long duration of hospital stay in a clinical service. Revista de la facultad de Ciencias Médicas, 2005; 62(1): 8-14.

13. MORAES A, et al. Caracterização de Pacientes Internados em Clínica Médica Segundo o Grau de Dependência do Cuidado de Enfermagem. In: Anais do Congresso Internacional de Humanidades \& Humanização em Saúde [Blucher Medical Proceedings, n.2, v.1]. São Paulo: Editora Blucher, 2014. ISSN 2357-7282

14. MOTTA CCR, et al. Perfil de internação de pessoas idosas em um hospital público. Rev Eletr Enf, 2010; 12(3): 471477.

15. RODRIGUES CC, RIBEIRO RCHM. Perfil epidemiológico dos pacientes atendidos na emergência de um hospital escola. Arq Ciênc Saúde, 2012; 19(2): 37-41.

16. RUFINO GP, et al. Avaliação de fatores determinantes do tempo de internação em clínica médica. Rev Bras Clin Med, 2012; 10(4): 291-297.

17. SILVA AMN. Fatores que contribuem para o tempo de internação prolongada no ambiente hospitalar. Journal of Research: Fundamental Care Online, 2014; 6(4): 1590-1600.

18. SILVA SA, et al. Fatores de atraso na alta hospitalar em hospitais de ensino. Rev Saúde Pública, 2014; 48(2): 314321.

19. SILVA RF, et al. Efeitos da hospitalização prolongada: o impacto da internação na vida do paciente e seus cuidadores. Saúde (Santa Maria), 2018; 44(3): 1-12.

20. SIQUEIRA AB, et al. Impacto funcional da internação hospitalar de pacientes idosos. Rev Saúde Publica, 2004; 38(5): 684-694.

21. SOUSA BA et al. Prevalência de infecção por tuberculose em funcionários de um hospital universitário. Revista Paraense de Medicina, 2021; 25(1): 1-8.

22. TIENSOLI SB, et al. Diagnóstico situacional: perfil sociodemográfico e clínico de pacientes internados em unidade de clínica médica. Revista Mineira de enfermagem, 2014; 18(3): 573-578.

23. WILLIAMS TA, et al. Long-term survival from intensive care: a review. Intensive Care Med, 2005; 31(10): $1306-1315$. 